\title{
Efficacy of L-Ornithine L-Aspartate for the prevention and Treatment of Hepatic Encephalopathy in Cirrhosis: An Update of the Evidence Base
}

\author{
Roger F Butterworth* \\ Department of Medicine, University of Montreal, Montreal Qc., Canada
}

${ }^{\star}$ Corresponding Author: Roger F Butterworth, Dept. of Medicine, University of Montreal, 45143 Cabot Trail, Englishtown, Nova Scotia, Canada; Tel: 19029292470; Email: rb@enceph.com

Received: November 14, 2019; Accepted: December 18, 2019; Published: December 20, 2019;

\begin{abstract}
The advent of well-established procedures for the determination of clinical trial quality based on risk of bias assessments has resulted insubstantial improvements in the quality of systematic reviews and meta-analyses relating to the assessment of Randomized Controlled Trials (RCTs) on the efficacy of treatments for a range of clinical conditions. In the current review, manual and electronic searches of databases using appropriate keywords were used to assess the evidence base for the use of L-ornithine L-aspartate (LOLA) for the prevention and treatment of Hepatic Encephalopathy (HE), a common neuropsychiatric complication of liver cirrhosis. Making use of current risk of bias techniques, seven systematic reviews with accompanying meta-analyses were identified in which the results of RCTs on the efficacy of LOLA for the treatment of HE were analyzed. A clear consensus of opinion was observed in support of the efficacy of LOLA for lowering of blood ammonia and for the concomitant improvement of mental status in patients with overt HE (OHE) and in five of the six meta-analyses in patients with minimal HE (MHE). Evidence in support of a beneficial effect of LOLA for the prevention of OHE in patients with cirrhosis was reported in a novel systematic review and meta-analysis involving the analysis of six RCTs in patients with cirrhosis and a range of clinical presentations where successful OHE prevention/prophylaxis was accompanied in all cases by significant reductions of blood ammonia. Both, intravenous and oral formulations of LOLA were found to be effective. Reduction in the progression of MHE to OHE was independently confirmed in a subsequent meta-analysis. Two systematic reviews with network meta-analyses compared the efficacy of LOLA to other available agents. Only treatment with LOLA or branched-chain amino acids (BCAAs) resulted in significant improvements in mental status and LOLA was judged to be the most effective agent with respect to clinical improvement and concomitant reduction of blood ammonia. In the case of MHE, rifaximin, lactulose and LOLA were equivalent in clinical efficacy and were each superior to probiotics. LOLA was superior to lactulose or probiotics for the prevention of episodes of OHE in patients with MHE compared to placebo/no treatment; rifaximin was ineffective in this regard.
\end{abstract}

Keywords: L-ornithine L-aspartate, LOLA, hepatic encephalopathy, clinical trials, RCTs, hyperammonemia, meta-analysis, systematic review, prevention, treatment, cirrhosis, sarcopenia, prophylaxis

\section{Introduction}

A variety of agents with the capacity to lower circulating ammonia represent the mainstay for the prevention and treatment of Hepatic Encephalopathy (HE) in patients with cirrhosis. Such agents include non-absorbable disaccharides, antibiotics, ammonia-sequestering compounds and metabolic intermediates related to the operation of the urea cycle. L-ornithine L-aspartate (LOLA) is a 1:1 stable salt of the naturally-occurring amino acids L-ornithine and L-aspartic acid. LOLA has well-established pharmacokinetic and pharmacodynamic properties and is available in either intravenous or oral formulations [1]. Increases in the use of LOLA for HE prevention and treatment of $\mathrm{HE}$ in patients with cirrhosis has resulted in a significant increase in the number of reports of the findings of RCTs on the efficacy of LOLA in this patient population and a number of reviews and metaanalyses on the subject have recently been published. For the current study, manual and electronic searches of databases using appropriate keywords were used to review and update the evidence base for the efficacy of LOLA for the prevention and treatment of HE in patients with cirrhosis. Particular attention was paid to assessment of the results of published RCTs, critical reviews, systematic reviews and metaanalyses in which the results of these trials were assessed. In addition, comparisons of the efficacy of LOLA compared to other currentlyavailable agents listed above has been addressed by assessment of the results of the results of two network meta-analyses. Since its discovery as an effective ammonia-lowering agent some 50 years ago [2], LOLA has been shown to act by virtue of the fact that one of its constituents, L-ornithine is a urea cycle substrate and both amino acids are substrates for transaminase reactions in multiple tissues including liver, brain and skeletal muscle leading to the production of glutamate, the obligate substrate for Glutamine Synthetase (GS). These two metabolic pathways, namelythe synthesis of urea (liver) and of glutamine (liver, brain, skeletal muscle) represent the major pathways for the elimination of excess ammonia under normal physiological conditions. In both acute and chronic liver failure, the metabolic capacity of the liver is severely compromised and urea and glutamine 
synthesis may fall to below $20 \%$ of normal values. This results in a spectacular increase in capacity of skeletal muscle to replace liver as the major ammonia-removal organ, a mechanism that results from increased expression of the gene coding for GS in muscle [3] resulting in increases in enzyme activities and increased glutamine synthesis. [4] In this way, it has been demonstrated that LOLA is effective for the treatment of muscle wasting (sarcopenia) in cirrhosis [5], a condition which, like HE is caused, at least in part, by the toxic actions of ammonia [6]. However, improvements in metabolic ammoniaremoval mechanisms are not the only ones where by LOLA treatment has beneficial effects on HE in cirrhosis. It has been demonstrated that LOLA has significant hepato-protective actions [7] mediated by the synthesis of the anti-oxidant glutathione (GSH) as well as the production of nitric oxide leading to improvements in hepatic microcirculation. $[7,8]$

\section{Efficacy of LOLA for the treatment of hyper am- monemia and $\mathrm{HE}$ in cirrhosis}

Beneficial effects of intravenous or oral formulations of LOLA have been reported in over 25 published Randomized Controlled Trials (RCTs). In most cases efficacy was defined in terms of LOLA's ammonia-lowering actions together with improvements in HE grade (for OHE) or psychometric test scores (for MHE). The last three years have seen the completion of several new trials and meta-analyses devoted to the assessment of the efficacy of LOLA for the treatment of HE in cirrhosis some of which have challenged or confirmed the results of earlier work. Consequently, the present review is an up-todate summary of the results of systematic reviews (with meta-analyses where available) of RCTs published through December 2019 on the efficacy of LOLA for the prevention and treatment of HE in patients with cirrhosis.

\subsection{Efficacy of LOLA for the treatment of HE in cirrhosis: early critical reviews of RCTs}

Results of clinical trials conducted in the 1980's and 1990's suggested that LOLA had the potential to lower blood ammonia and decrease the severity of HE. In order to assess this possibility two critical analyses were undertaken. In the first analysis, a search of indexed medical journals in which the results of RCTs were described in patients with cirrhosis and HE treated with LOLA. Four RCTs published during the period 1993-2000 for a total of 217 patients met inclusion criteria two of which made use of a parallel group design that included patients with MHE and two trials using a crossover design and patients with low-grade (I or II) OHE. [9] LOLA treatment led to lowering of blood ammonia [9] in patients with HE when compared to placebo using either intravenous (iv) or oral formulation of LOLA. This lowering of blood ammonia was accompanied by improvements in psychometric test scores but was not uniformly accompanied by improvements in mental status measured using the PSE Index procedure [9] (Table 1).

Table 1. Critical reviews of RCTs for LOLA treatment of HE in cirrhosis

\begin{tabular}{|c|c|c|c|c|c|c|}
\hline Study ID & Year & $\begin{array}{c}\text { No of } \\
\text { trials }\end{array}$ & $\begin{array}{c}\text { No of } \\
\text { patients }\end{array}$ & Type of HE & $\begin{array}{c}\text { Ammonia- } \\
\text { lowering }\end{array}$ & Outcome parameters \\
\hline Perez Hernandez JL & 2011 & 5 & 623 & MHE, OHE & Yes & $\begin{array}{l}\text { Improvement of mental } \\
\text { status, Ammonia, } \\
\text { Hospitalization time }\end{array}$ \\
\hline
\end{tabular}

Summary

Database searches of controlled trials identified six meeting the inclusion criteria for a total of 623 patients. LOLA infusions let to improvement in neuropsychiatric status, decreased serum ammonia with minimal adverse events.

\begin{tabular}{|l|l|l|l|l|l|l|}
\hline Soarez PC & 2009 & 4 & 217 & MHE, OHE & Yes & $\begin{array}{l}\text { Ammonia; Improvement in } \\
\text { psychometric test }\end{array}$ \\
\hline
\end{tabular}

Summary

Database searches of controlled clinical trials (English language) yielded four RCT's with a total of 217 patients for inclusion in the analysis. LOLA (iv or oral) treatment resulted in reduced hyperammonemia compared to placebo and improved psychometric test scores. Small trial/patient numbers and low methodological quality limited beneficial effect in patients with OHE.

In a second critical analysis published two years later, searches were made of RCTs that were again published in indexed journals as well as in Medline, Cochrane and PubMed databases in which the efficacy of ivLOLA was assessed in patients with cirrhosis and HE. Six trials met inclusion criteria for a total of 623 patients 422 of which had cirrhosis while the remainder had acute liver failure [10].Trial quality was assessed using the Jadad Composite scale. [11] Venous ammonia concentrations decreased in the LOLA treatment group compared to placebo and these decreases were accompanied by significant improvements in the stage of $\mathrm{HE}$ assessed by West Haven criteria (Table 1).

\subsection{Efficacy of LOLA for the treatment of HE in cirrhosis: systematic reviews of RCTs with meta-analyses}

Results of seven systematic reviews each accompanied by metaanalysis of the results of RCTs on the efficacy of LOLA for the efficacy of treatment of MHE/OHE in patients with cirrhosis have been completed and published in the last 20 years starting with an in house 
Butterworth RF (2019) Efficacy of L-Ornithine L-Aspartate for the prevention and Treatment of Hepatic Encephalopathy in Cirrhosis: An Update of the Evidence Base

analysis of five trials from Merz Pharmaceuticals (Germany) [12] Subsequent analyses by investigators from China. [13-15] Europe $[16,17]$ Canada [18] and India [19] followed involving up to 36 trials and 2377 patients with cirrhosis and HE. Summaries of the numbers of RCTs, patients, year, type of HE, outcome parameters, publication reference and short synopsis of the major findings are provided in Table 2.

Table 2. Systematic reviews with meta-analysis of RCTs for LOLA treatment of HE in cirrhosis

\begin{tabular}{|c|c|c|c|c|c|c|}
\hline Study ID & Year & $\begin{array}{c}\text { No of } \\
\text { trials }\end{array}$ & $\begin{array}{c}\text { No of } \\
\text { patients }\end{array}$ & Type of HE & $\begin{array}{c}\text { Ammonia- } \\
\text { lowering }\end{array}$ & Outcome parameters \\
\hline Butterworth RF & 2018 & 10 & 884 & MHE, OHE & Yes & $\begin{array}{l}\text { Benefit for OHE; MHE iv/ } \\
\text { oral, NH3-lowering }\end{array}$ \\
\hline
\end{tabular}

Summary

Electronic and manual searches were made of databases to identify RCTs for inclusion. Ten RCTs were included for a total of 884 patients with cirrhosis and HE Random effects model used to express pooled risk ratio (RR) or Mean difference (MD). Both intravenous and oral formulations of LOLA found to be effective for lowering of blood ammonia [MD: $-17.5 \mu \mathrm{mol} / 1(-27.73,-7.26)] \mathrm{p}<0.0008$ and improvement of mental state for patients with MHE [RR: $2.15,95 \%$ CI: 1.48-3.14) p<0.0001)] or OHE [RR: 1.19, 95\% CI: $1.01-1.39, \mathrm{p}<0.03]$. Oral LOLA was particularly effective for treatment of MHE.

\begin{tabular}{|l|c|c|c|c|c|c|c|}
\hline Goh ET & 2018 & 22 & 1375 & MHE, OHE & Yes & $\begin{array}{l}\text { Benefit for OHE/MHE, NH3- } \\
\text { lowering }\end{array}$ & $\begin{array}{l}\text { Cochrane Database Syst Rev. } \\
2018 ; 5: C D 012410\end{array}$ \\
\hline
\end{tabular}

Summary

Electronic and manual searches of databases, conference proceedings and correspondence with investigators and pharmaceutical companies yielded 22 RCTs involving 1375 patients with cirrhosis and HE or risk of development of HE for which outcome data was available. LOLA had a beneficial effect on HE compared to placebo/no intervention for all trials [RR: $0.70,95 \%$ CI: $0.59-0.88$ ] but evidence was judged to be very low quality leading investigators to conclude that outcomes were uncertain. However, subsequent sub-group analyses of completed RCTs and/or RCTs with findings published as full papers demonstrated significant improvements in mental state: 12 completed trials, 994 patients : RR:0.63, 95\% CI: 0.48-0.83, p <0.001], 12 published trials, 1032 patients: RR:0.65,95\% CI: $0.50-0.85$, $\mathrm{p}<0.0017]$. Both iv and oral formulations appeared to be effective in this analysis.

\begin{tabular}{|l|c|c|c|c|c|l|}
\hline Bai M & 2013 & 8 & 646 & MHE, OHE & Yes & $\begin{array}{l}\text { Benefit for OHE; MHE, } \\
\text { NH3-lowering }\end{array}$ \\
\hline
\end{tabular}

Summary

Searches of databases revealed 8 RCTs that assessed the efficacy of LOLA for treatment of HE in 646 patients with cirrhosis. LOLA was significantly more effective than placebo/no intervention for improvement in all types of HE [RR: 1.49, 95\% CI: 1.10-2.01, p $<0.01]$ as well as for patients with OHE or MHE when analysed separately. These improvements were accompanied by significant reductions in fasting blood ammonia [MD: $-18.26,95 \%$ CI: $-26.96-9.56, \mathrm{p}<0.01]$.

\begin{tabular}{|l|c|c|c|c|c|l|l}
\hline Hu Wei & 2012 & 6 & 432 & MHE, OHE & Yes & $\begin{array}{l}\text { Serum ammonia, NCT-A, } \\
\text { Clinical remission rate }\end{array}$ & $\begin{array}{l}\text { Chin J Evidence-based Med 2012; } \\
(12) 7: 799-803\end{array}$ \\
\hline
\end{tabular}

Summary

Database searches of RCT's of LOLA (iv or oral) for treatment of HE in cirrhosis yielded six placebo-controlled trials and 432 patients. LOLA significantly reduced serum ammonia $(\mathrm{p}<0.0001)$, improved NCT-A scores $(\mathrm{p}<0.0001)$ and clinical remission rates $(\mathrm{p}<0.01)$.

\begin{tabular}{|l|c|c|c|c|c|c|c}
\hline Jiang Q & 2009 & 3 & 212 & $\begin{array}{l}\text { Chronic OHE } \\
(1,2)\end{array}$ & Yes & $\begin{array}{l}\text { Benefit for OHE not MHE } \\
\text { Jan;24 (1):9-14 }\end{array}$ \\
\hline
\end{tabular}

Summary

Searches of electronic databases yielded 3 RCTs of 212 patients of sufficiently high quality (assessed by Jadad score) for inclusion in the analysis. LOLA significantly improved HE scores [RR: 1.89, 95\% CI: 1.32-2.71, p<0.0005]. Subgroup analysis revealed significant efficacy of LOLA compared to placebo (2 trials) or lactulose (1 trial) in patients with grades I or II HE but not in patients with MHE.

\begin{tabular}{|l|c|c|c|c|c|c|}
\hline Delcker M & 2000 & 5 & 246 & MHE, OHE & Yes & $\begin{array}{l}\text { Ammonia, improvement of } \\
\text { mental state, psychometric } \\
\text { test scores }\end{array}$ \\
\hline
\end{tabular}

Summary

This review with meta-analysis was the first conducted by the manufacturers of LOLA and consisted of assessment of the efficacy of iv LOLA in 5 RCTs versus placebo. Two of the trials were subsequently published. Treatment with LOLA for 7 days resulted in significant improvements of NCT-A scores and mental state as a function of the lowering of blood ammonia. 
Results were, in general, remarkably consistent with all seven meta-analyses showing evidence of improvements of mental state in patients with MHE or OHE [12-19] that was accompanied by lowering of blood ammonia in all cases. When assessed separately, either intravenous or oral formulations of LOLA were found to be effective for the treatment of HE [15-18] However, occasional inconsistencies were noted and this was attributed to differences in experimental design, inclusion/exclusion criteria or methodology used for the determination of mental state. For example, in one earlier study the patient population included cirrhotics as well as patients with Acute Liver Failure (ALF) [14]; the pathophysiology and treatment goals for the two conditions are quite distinct. In a second study, LOLA treatment was found to be ineffective for improvement of psychometric test scores in patients with MHE [13] but was found to be effective in all subsequent analyses in which this was addressed $[15,18]$. One possible explanation likely relates to the differences in the nature of the psychometric test procedures used in these analyses (e.g. use of the outdated PSE Index scoring system in one analysis[13] versus multiple well-established psychometric testing procedures such as NCT-A, B and PHES in the others). It is important to note that there are also areas of investigation relating to the efficacy of LOLA for the treatment of $\mathrm{HE}$ in cirrhosis that have been largely omitted from these earlier analyses. For example, few of these analyses investigated the possible beneficial effects of LOLA on ammonia lowering or mental state improvement in patients with higher grades (III and IV) of HE $[12,14]$.In addition, there are no published systematic reviews and/or meta-analyses relating to the efficacy of LOLA for the prevention and treatment of HE in cirrhosis in which the new system of classification of HE (i.e. Covert, Overt grades II,III,IV) was employed. The advent of well-established procedures for the determination of trial quality based on risk of bias assessments has led to significant improvements in the quality of subsequent systematic reviews with meta-analyses. Such procedures include use of the Jadad Composite Scoring system [11] and, more recently, by the Cochrane Handbook for Systematic Reviews and Interventions[20]. Combinations of the two systems have also been employed[18,21].These systems used for assessment of risk of bias of each RCT take into account sequence generation during randomization, allocated sequence concealment, blinding of participants and personnel and completeness of outcome data[11,20]. In the first systematic review with meta-analysis undertaken under the above guidelines, Bai and co-workers searched manual and/ or electronic databases to reveal eight RCTs with 646 patients with cirrhosis and OHE or MHE in which the efficacy of LOLA (iv or oral formulations) was compared to placebo/no intervention [15]. Study endpoints were improvement in $\mathrm{HE}$ and lowering of blood ammonia. LOLA was significantly more effective than placebo/no intervention for improvement of all types of HE with RR: 1.49, 95\% CI:1.10-2.01, $\mathrm{p}<0.01$ by Random Effects model. Significant benefit was also recorded for improvement of OHE with RR: 1.33, 95\% CI: 1.04-1.69, p $<0.02$ by Random Effects model as well as for MHE with RR: 2.25 , 95\%CI: $1.33-2.82, \mathrm{p}<0.01$ by Fixed Effects model. Reduction of fasting blood ammonia significantly favored LOLA over placebo/no intervention with $\mathrm{p}<0.01$. In a subsequent systematic review with meta-analysis, 10 RCTs with 884 patients with cirrhosis and HE satisfied inclusion criteria. [18] Study quality and risk of bias were assessed using the
Jadad Composite scale combined with the Cochrane Scoring Tool and the Random Effects Model was employed to express pooled Risk Ratio (RR) or Mean Difference (MD) with associated 95\% Confidence Intervals (CI). Comparison with placebo/no intervention control data, LOLA was found to be significantly more effective for improvement of mental scores in all types of HE [RR: $1.36,95 \%$ CI: $1.10-1.69$, $\mathrm{p}<0.005$ ] as well as in patients with OHE [RR: 1.19, 95\% CI: 1.01-1.39, $\mathrm{p}<0.03$ ] or MHE [RR: 2.15, 95\% CI: $1.48-3.14, \mathrm{p}<0.0001]$. LOLA treatment resulted in significant lowering of blood ammonia in these patient groups [MD: $-17.5 \mathrm{umol} / \mathrm{L}, 95 \% \mathrm{CI}:-27.73$ to $-7.26, \mathrm{p}<0.008$ ] The oral formulation of LOLA was found to be particularly effective for the treatment of patients with MHE. A similar systematic review with meta-analysis identified 15 RCTs and 1023 patients with cirrhosis and HE in which treatment with LOLA resulted in significant benefit for subgroups of patients with acute episodes of HE or with chronic HE but not in patients with MHE in an initial analysis of the data [16]. One year later, a large number of additional trials were added to this particular investigation giving a total of 36 RCTs with 2377 patients. Regrettably, data for the majority of these additional trials was found to be seriously lacking due to early trial abandonment as well as incomplete information required for assessment of risk of bias and trial outcomes leading the investigators to rate them as very low quality and to express uncertainty in the reliability of the findings [17]. Fortunately, there was a sufficient number of completed and/or published trials in this study to permit subgroup analysis in relation to the efficacy of LOLA for the treatment of HE. The relevant data was:

For completed trials [12 trials, 994 patients, RR: 0.63, 95\% CI: $0.48-0.83, \mathrm{p}<0.001]$

For published trials [12 trials, 1026 patients, RR: $0.65,95 \%$ CI: $0.50-0.85, \mathrm{p}<0.00017]$

These findings confirm those of three previous systematic reviews with meta-analysis dedicated to the assessment of the efficacy of LOLA for the treatment of OHE or MHE [15-19]

\subsection{Efficacy of ammonia scavengers other than LOLA for the treatment of HE in cirrhosis: results of a meta-analysis}

Searches of on-line databases and clinical trials registries yielded 11 RCTs that met inclusion criteria. [22] Meta-analysis using Risk Ratios (RR) or Mean Differences (MD) with 95\% CI was performed with bias assessment. By design, the agents selected for this analysis did not include LOLA even though, as demonstrated and discussed in section 2.2 (above), it is the best-established agent currently employed clinically for the treatment of HE that specifically targets ammonia. Selection of most of these agents was undoubtedly inspired by their successful use for ammonia-lowering in cases of acute or chronic hyperammonemia associated with congenital deficiencies of urea cycle enzymes. Such agents included sodium benzoate (three trials), glycerol phenylbutyrate (one trial) and ornithine phenylacetate (two trials) in addition to AST-120 (two trials) and polyethylene glycol (three trials) for a total of 499 patients receiving test substance versus 444 receiving placebo or lactulose. Eight of the eleven trials were assessed as very low quality having high risks of bias. [22] Not surprisingly, significant reductions of blood ammonia were observed in placebo- 
Butterworth RF (2019) Efficacy of L-Ornithine L-Aspartate for the prevention and Treatment of Hepatic Encephalopathy in Cirrhosis: An Update of the Evidence Base

controlled trials of sodium benzoate, glycerol phenylbutyrate and ornithine phenylacetate but with no observable effects of the latter substance on HE grade. Sodium benzoate, polyethylene glycoland AST-120 treatments failed to show significant improvements in HE grade compared to lactulose. These results led the authors to conclude that, although there was potential for reduction of blood ammonia by these agents, their effects on clinical outcome remain uncertain. This appeared to be primarily due to the low quality of the trials selected for the analysis. [22]

\subsection{Efficacy of LOLA for OHE prevention and prophylaxis: systematic review with meta-analysis}

There is a paucity of available published reports of systematic reviews with meta-analysis of RCTs dedicated to the evaluation of the efficacy of LOLA for the prevention of HE in patients with cirrhosis. Sporadic reports are limited in number to sub-groups of patients but results so far have been inconsistent $[16,19]$ largely due to small trial numbers and low patient enrollment in addition to very low quality of the data leading investigators to conclude that the evidence for prevention of either OHE or MHE was uncertain. [17] Consequently a new systematic review with meta-analysis was undertaken to review the evidence base in support of a beneficial effect of LOLA for the prevention/prophylaxis of OHE in patients with cirrhosis. Electronic and manual searches identified 6 RCTs that met inclusion criteria for a total of 384 patients. [21] Five of the six trials were considered to be high quality with low risk of bias by Jadad-Cochrane criteria. LOLA treatment led to a significant reduction in the rate of progression of MHE to OHE compared to placebo/no intervention (three trials) with RR: $0.23,95 \%$ CI: $0.07-0.73, \mathrm{p}<0.01$. LOLA treatment was also effective for secondary OHE prophylaxis, for primary OHE prophylaxis following gastrointestinal bleeding (one trial) and for post-TIPSS prophylaxis (one trial). Successful OHE prevention/prophylaxis was accompanied by significant reductions of blood ammonia and either iv or oral formulations of LOLA appeared to be effective for the slowing of progression of MHE to OHE. The effectiveness of LOLA versus placebo for reduction of the progression of MHE to OHE in patients with cirrhosis was independently confirmed in a subsequent meta-analysis. [19]

Table 3. Systematic reviews with meta-analysis of RCTs for OHE prevention/prophylaxis by LOLA

\begin{tabular}{|c|c|c|c|c|c|c|c|}
\hline Butterworth RF & 2019 & 6 & 384 & MHE, OHE & No & $\begin{array}{l}\text { OHE prevention; progression } \\
\text { from MHE to OHE }\end{array}$ & $\begin{array}{l}\text { Metab Brain Dis 2019. https://doi. } \\
\text { org/10.1007/s11011-019-00463-8 }\end{array}$ \\
\hline \multicolumn{8}{|c|}{$\begin{array}{l}\text { Summary } \\
\text { Electronic and manual searches together with pre-established inclusion/exclusion criteria revealed } 6 \text { RCTs for a total of } 384 \text { patients with cirrhosis at risk for development of OHE. } \\
\text { Treatment with iv or oral LOLA led to significant reductions in the risk of progression to OHE in patients with MHE [ } 3 \text { trials with RR: } 0.23,95 \% \text { CI:0.07-0.73) p, } 0.01 \text {. LOLA was } \\
\text { also effective for secondary OHE prophylaxis [ } 1 \text { trial with RR: } 0.389,95 \% \text { CI: } 0.174-0.870, p<0.002] \text { and for OHE prophylaxis following acute variceal bleeding [ } 1 \text { trial with RR: } \\
0.42,95 \% \text { CI: } 0.16-0.98, p<0.03 \text { ] and for OHE prophylaxis post-TIPSS [ } 1 \text { trial with OR: } 0.20,95 \% \text { CI: } 0.06-0.88, p<0.03 \text { ] OHE prevention/prophylaxis was accompanied by signif- } \\
\text { icant reductions of blood ammonia. Both iv and oral formulations of LOLA were effective. }\end{array}$} \\
\hline
\end{tabular}

Figure 1a. Forest Plot for the efficacy of LOLA versus placebo/no intervention for the prevention of progression of MHE to OHE (Abid et al. 2011; Mittal et al. 2011; Alvares-da-Silva et al. 2014), secondary OHE prophylaxis (Varakanahalli et al. 2018), primary OHE prophylaxis(Higuera-de-la-Tijera et al. 2018) or post-TIPSS OHE prophylaxis (Bai et al. 2014)

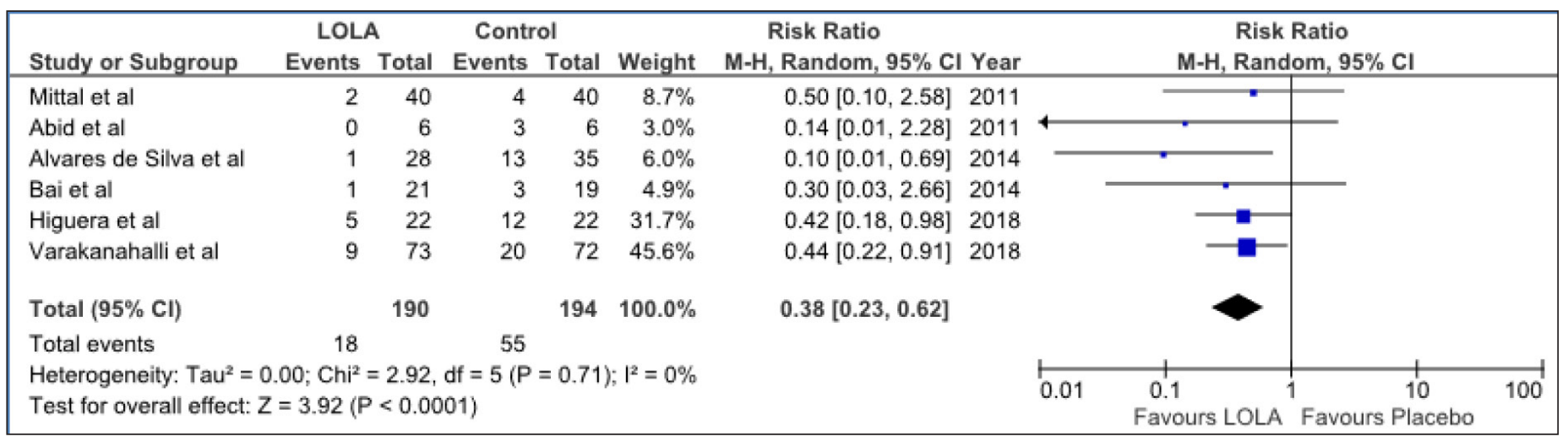

Figure 1b. Forest plot for the efficacy of LOLA versus placebo/no intervention for the prevention of progression from MHE to OHE in patients with cirrhosis

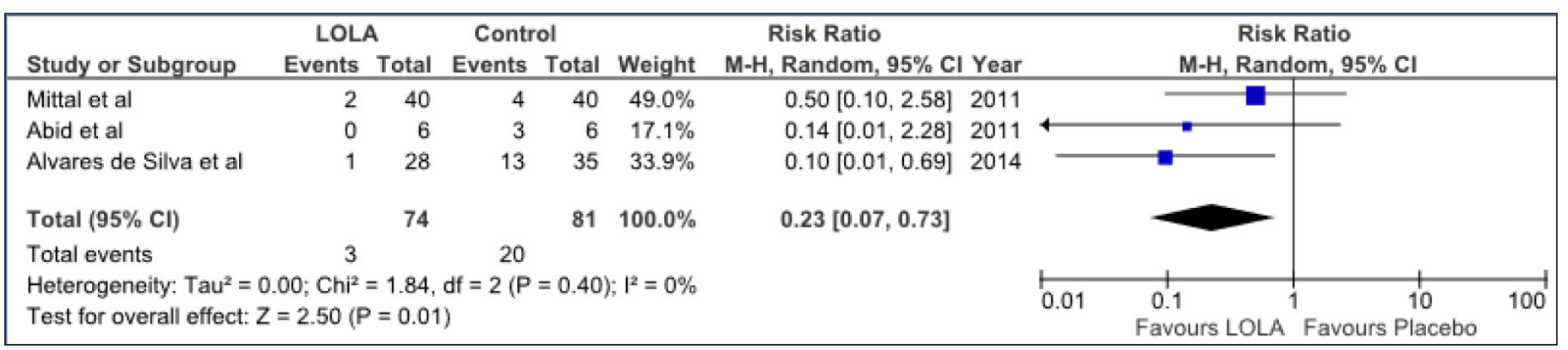




\section{Efficacy of LOLA compared to other currently-avail- able agents for the treatment of HE in cirrhosis: net- work meta-analyses}

RCTs directly comparing the efficacy of LOLA with other available agents such as non-absorbable disaccharides, antibiotics and probiotics have consistently shown that LOLA is equivalent and, in some cases, superior to these alternatives. For example, in an RCT published in 2006, patients randomized to lactulose or LOLA manifested comparable decreases of blood ammonia but only patients in the LOLA arm of the trial showed improvements in psychometric test scores, mental state grade, asterixis grade or EEG. [23] These observations were followed by two systematic reviews with network meta-analyses in which the efficacy of LOLA for the treatment of HE in patients with cirrhosis was compared to other available agents. The first analysis addressed the treatment of OHE [23], the second one focused on the treatment of MHE and on the progression from MHE to OHE [19].

Table 4. Network meta-analyses of RCTs comparing efficacy of LOLA versus other available agents for treatment of HE in cirrhosis

\begin{tabular}{|c|c|c|c|c|c|c|c|}
\hline Study ID & Year & $\begin{array}{l}\text { No of } \\
\text { trials }\end{array}$ & $\begin{array}{c}\text { No of } \\
\text { patients }\end{array}$ & Type of HE & $\begin{array}{l}\text { Ammonia- } \\
\text { lowering }\end{array}$ & Outcome parameters & Reference \\
\hline Dhiman RK & 2019 & 25 & 1563 & MHE, OHE & Yes & $\begin{array}{l}\text { Comparable efficacy of } \\
\text { LOLA for reversal of MHE; } \\
\text { Prevention of OHE }\end{array}$ & $\begin{array}{l}\text { Clin Gastroenterol Hepatol. 2019 Aug 30. } \\
\text { pii: S1542-565(19) 30969-3. doi: } 10.1016 / \mathrm{j} \text {. } \\
\text { cgh.2019.08.047 }\end{array}$ \\
\hline \multicolumn{8}{|c|}{ Summary } \\
\hline \multicolumn{8}{|c|}{$\begin{array}{l}\text { A systematic search of databases for RCTs evaluating treatments for MHE and prevention of deterioration to OHE resulted in a Network meta-analysis with surface under } \\
\text { cumulated ranking (SUCRA) for rifaximin, lactulose, probiotics, probiotics + lactulose or LOLA compared to placebo/no treatment. Twenty five trials identified with } 1563 \\
\text { patients with cirrhosis and MHE. LOLA was effective for reversal of MHE [ OR: } 4.45,95 \% \text { PrI: } 2.67-7.42 \text {, SUCRA: } 47.2 \% \text {, moderate quality] compared to placebo/no } \\
\text { treatment and LOLA and lactulose were most effective for preventing episodes of OHE. Comparative analysis revealed no superiority between other agents and LOLA. }\end{array}$} \\
\hline Zhu GQ & 2015 & 20 & 1.007 & OHE & No & LOLA $=$ BCAA $>$ LAC $>$ NEO & Aliment Pharmacol Ther 2015; 41: 624-635 \\
\hline \multicolumn{8}{|c|}{ Summary } \\
\hline \multicolumn{8}{|c|}{$\begin{array}{l}\text { Literature searches including databases revealed } 20 \text { eligible RCTs for inclusion in this Network meta-analysis comparing efficacy of LOLA to that of BCAAs, non-absorbable } \\
\text { disaccharides and neomycin compared to observation. The analysis combined direct and indirect evidence to estimate Odds Ratio (OR) and mean difference (MD) between } \\
\text { treatments. Compared to observation, only LOLA [OR: } 3.71, \mathrm{p}<0.001] \text { and BCAAs [OR: } 3.37, \mathrm{p}<0.001] \text { improved clinical efficacy significantly. There was a trend suggesting } \\
\text { that LOLA was the most effective intervention with respect to clinical improvement [OR" } 1.10] \text {. LOLA treatment resulted in a significant reduction in blood ammonia [MD:- } \\
20.18,95 \% \text { CI: }-40.12-0.27 \text { ]. }\end{array}$} \\
\hline
\end{tabular}

\subsection{Network meta-analysis: treatment of OHE by LOLA vs other agents}

Electronic and manual searches of key databases yielded 20 RCTs that satisfied inclusion criteria for 1007 patients with cirrhosis and OHE who were treated with non-absorbable disaccharides, neomycin, rifaximin, LOLA or BCAAs versus observation only. Network metaanalysis combined direct and indirect evidence to obtain Odds Ratios (ORs) or Mean Differences (MDs) between treatments based on clinical outcomes. [23] Compared to observation only, treatment with LOLA [OR: 3.71, $\mathrm{p}<0.001]$ or BCAAs [OR: 3.37, $\mathrm{p}<0.001]$ resulted in significant improvements in clinical efficacy. It was also concluded that LOLA had the potential to be the most effective intervention with respect to clinical improvement [OR: 1.10], rifaximin [OR: 1.31], non-absorbable disaccharides [OR: 2.75] or neomycin [OR: 2.22]. Moreover, LOLA treatment resulted in a significant reduction in blood ammonia [MD: $-20.18,95 \%$ CI: -40.12 to -0.27 ]compared to observation alone.

\subsection{Network meta-analysis: treatment of MHE by LOLA vs other agents}

Search of databases for RCTs evaluating available treatments for MHE in patients with cirrhosis yielded 25 trials for 1563 patients that satisfied inclusion criteria. There were two primary outcomes, namely reversal of MHE and prevention of progression from MHE to OHE using meta-analysis followed by Network meta-analysis. SUCRA was employed to pool direct and indirect estimates and to rank the various treatments.

Rifaximin, lactulose and LOLA were equivalent in efficacy and were each superior to probiotics with or without lactulose shown below:

- Rifaximin [OR:7.53, 95\% PrI: 4.45-12.73, SUCRA: 89.2\%; moderate quality]

- Lactulose [OR: 5.39, 95\% PrI: 3.60-8.07, SUCRA: 67.2\%; moderate quality]

- LOLA [OR: 4.45, 95\% PrI: 2.67-7.42, SUCRA: 47.2\%; moderate quality]

- Probiotics+ lactulose [OR: 4.66, 95\% PrI: 1.90-11.39, SUCRA: $52.4 \%$; low quality]

- Probiotics [OR: 3.89, 95\%PrI: 2.52-6.02, SUCRA: 34.1\%; low quality]

LOLA was superior to lactulose or probiotics for the prevention of episodes of OHE in patients with MHE compared to placebo/no treatment as shown below:

- LOLA [OR: 0.19, 95\% PrI: 0.04-0.91, SUCRA: 75.1\%; moderate quality] 
- Lactulose [OR: 0.22, 95\% PrI: 0.09-0.52, SUCRA: 73.9\%; moderate quality]

- Probiotics [OR: 0.27, 95\% PrI: 0.11-0.62, SUCRA: 59.6\%; low quality.

Rifaximin, on the other hand, was ineffective for OHE prevention [19].

\section{Conclusion}

The advent of well-established procedures for the determination of trial quality based on risk of bias assessments such as the Jadad Composite Scoring system followed, more recently, by the Cochrane Handbook for Systematic Reviews and Interventions has resulted in significant improvements in the quality of systematic reviews and meta-analyses of clinical trials. Making use of such procedures, seven systematic reviews with accompanying meta-analysis were published in the last 20 years all of which focused on the analysis of the results of RCTs on the efficacy of LOLA for the efficacy of treatment of MHE and/or OHE in patients with cirrhosis. An initial in-house metaanalysis by Merz Pharmaceuticals (Germany) published in 2000 was followed by systematic reviews and meta-analyses conducted by international investigators from China, Europe, Canada and India. Analysis of the findings from these seven meta-analyses reveals a clear consensus of opinion in support of the efficacy of LOLA for lowering of blood ammonia and for the concomitant improvement of mental status in patients with cirrhosis and OHE in all cases. For MHE, results from five of the six meta-analyses in which it was assessed also yielded significant positive results. A recent meta-analysis assessing the efficacy of other agents with the demonstrated capacity to lower blood ammonia in a range of clinical settings confirmed the lowering of blood ammonia by most agents. However, effects on HE severity were inconsistent leading the investigators to question the quality of the studies. By design, LOLA had not been included in the list of agents assessed in this analysis. The evidence in support of a beneficial effect of LOLA for the prevention of OHE in patients with cirrhosis was reviewed in a novel systematic review and meta-analysis involving six RCTs for a total of 384 patients in a range of clinical presentations. LOLA treatment led to a significant reduction in progression of MHE to OHE compared to placebo/no intervention (three trials) and LOLA treatment was also effective for secondary OHE prophylaxis (one trial), primary OHE prophylaxis following variceal bleeding (one trial) and for post-TIPSS prophylaxis (one trial). Successful OHE prevention/prophylaxis was accompanied in all cases by significant reductions of blood ammonia and either iv or oral formulations of LOLA appeared to be effective for the slowing of progression of MHE to OHE. The effectiveness of LOLA versus placebo for reduction of the progression of MHE to OHE in patients with cirrhosis [20] was independently confirmed in a subsequent meta-analysis. The efficacy of LOLA was compared to other currently-available agents for the treatment of HE in cirrhosis using the technique of network metaanalyses. Two systematic reviews with network meta-analyses have been published in which the efficacy of LOLA for the treatment of HE in patients with cirrhosis was compared to other available agents. The first analysis addressed the treatment of OHE; the second one focused on the treatment of MHE as well as the progression from MHE to OHE.

For treatment of OHE, only treatment with LOLA or BCAAs resulted in significant improvements in clinical efficacy. It was also concluded that LOLA had the potential to be the most effective intervention with respect to clinical improvement and LOLA treatment resulted in concomitant reductions of blood ammonia. For the treatment of MHE, rifaximin, lactulose and LOLA were found to be equivalent in efficacy and were each superior to probiotics with or without lactulose. LOLA was superior to lactulose or probiotics for the prevention of episodes of OHE in patients with MHE compared to placebo/no treatment. Rifaximin, on the other hand, was found to be ineffective for OHE prevention.

\section{References}

1. Kircheis G and Lüth S (2019) Pharmacokinetic and Pharmacodynamic Properties of L-Ornithine L-Aspartate (LOLA) in Hepatic Encephalopathy. Drugs 79: 23-29. [crossref]

2. Butterworth RF (2019) L-Ornithine L-Aspartate (LOLA) for the Treatment of Hepatic Encephalopathy in Cirrhosis: Novel Insights and Translation to the Clinic. Drugs 79: 1-3. [crossref]

3. Desjardins P, Rao KV, Michalak A, Rose C, Butterworth RF (1999) Effect of portacaval anastomosis on glutamine synthetase protein and gene expression in brain, liver and skeletal muscle. Metab Brain Dis 14: 273-80. [crossref]

4. Chatauret N, Desjardins P, Zwingmann C, Rose C, Rao KV et.al (2006) Direct molecular and spectroscopic evidence for increased ammonia removal capacity of skeletal muscle in acute liver failure. J Hepatol 44: 1083-8. [crossref]

5. Butterworth RF (2019) L-Ornithine L-Aspartate for the Treatment of Sarcopenia in Chronic Liver Disease: The Taming of a Vicious Cycle. Can J Gastroenterol Hepatol : 8182195 [crossref]

6. Kumar A, Davuluri G, Silva RNE, Engelen MPKJ, Ten Have GAM et.al (2017) Ammonia lowering reverses sarcopenia of cirrhosis by restoring skeletal muscle proteostasis. Hepatology 65: 2045-2058. [crossref]

7. Butterworth RF (2019) L-Ornithine L-Aspartate: Multimodal Therapeutic Agent for Hyperammonemia and Hepatic Encephalopathy in Cirrhosis. J Pharmacol Pharm Res 2: 1-7.

8. Ijaz S, Yang W, Winslet MC, Seifalian AM (2005) The role of nitric oxide in the modulation ofhepatic microcirculation and tissue oxygenation in an experimental animal model of hepatic steatosis. Microvasc Res 70: 129-136. [crossref]

9. Pérez Hernández JL, Higuera de la Tijera F, Serralde-Zúñiga AE, Abdo Francis JM (2011) Critical analysis of studies evaluating the efficacy of infusion of L-ornithine L-aspartate in clinical hepatic encephalopathy in patients with liver failure. Ann Hepatol 2: 66-69. [crossref]

10. Soárez PC, Oliveira AC, Padovan J, Parise ER, Ferraz MB (2009) A critical analysis of studies assessing L-ornithine-L-aspartate (LOLA) in hepatic encephalopathy treatment. Arq Gastroenterol 46: 241-247. [crossref]

11. Jadad AR1, Moore RA, Carroll D, Jenkinson C, Reynolds DJ et al. (1996) Assessing the quality of reports of randomized clinical trials: is blinding necessary? Control Clin Trials 17: 1-12. [crossref]

12. Delcker M, Jalan R, Schumacher M, Comes G (2000) L-ornithine-L-aspartate vs placebo in the treatment of hepatic encephalopathy: A meta-analysis of randomised placebo-controlled trials using individual data. Hepatology 32: 604. (abstract)

13. Jiang Q, Jiang XH, Zheng MH, Chen YP (2009) L-Ornithine-1-aspartate in the management of hepatic encephalopathy: a meta-analysis. $J$ Gastroenterol Hepatol 24: 9-14. [crossref]

14. Hu Weiand Tang SH (2012) Efficacy of L-ornithine-L-aspartate in the Treatment of Hepatic Encephalopathy: A Systematic Review. Chin J Evidence-based Med (12): 799-803. (article in Chinese)

15. Bai M, Yang Z, Qi X, Fan D, Han G (2013) L-ornithine-1-aspartate for hepatic encephalopathy in patients with cirrhosis: a meta-analysis of randomized controlled trials. J Gastroenterol Hepatol 28: 783-92. [crossref]

16. Goh ET, Stokes CS, Vilstrup H, Gluud LL, Morgan MY (2017) L-ornithine L-aspartate for hepatic encephalopathy: a systematic review with meta-analyses of randomised controlled trials. J Hepatol 66: 131 (abstract)

17. Goh ET, Stokes CS, Sidhu SS, Vilstrup H, Gluud LL et.al (2018) L-ornithine L-aspartate for prevention and treatment of hepatic encephalopathy in people with cirrhosis. Cochrane Database Syst Rev 15: 5. [crossref] 
Butterworth RF (2019) Efficacy of L-Ornithine L-Aspartate for the prevention and Treatment of Hepatic Encephalopathy in Cirrhosis: An Update of the Evidence Base

18. Butterworth RF, Kircheis G, Hilger N, McPhail MJW (2018) Efficacy of l-ornithinelaspartate for the treatment of hepatic encephalopathy and hyperammonemia incirrhosis: systematic review and meta-analysis of randomized controlled trials. $J$ Clin Exp Hepatol 8: 301-13. [crossref]

19. Dhiman RK, Thumburu KK, Verma N, Chopra M, Rathi S et.al (2019) Indian National Association for Study of Liver (INASL) Hepatic Encephalopathy Study Group (IHESG). Comparative Efficacy of Treatment Options for Minimal Hepatic Encephalopathy: A Systematic Review \& Network Meta-analysis. Clin Gastroenterol Hepatol 19: 30969-3.

20. Higgins JPT, Green S (2011) Cochrane Handbook for Systematic Review of Interventions Version 5.1.0 [updated March 2011]. The Cochrane Collaboration.
21. Butterworth RF (2019) Beneficial effects of L-ornithine L-aspartate for prevention of overt hepatic encephalopathy in patients with cirrhosis: a systematic review with meta-analysis. Metab Brain Dis [crossref]

22. Zacharias HD, Zacharias AP, Gluud LL, Morgan MY (2019) Pharmacotherapies that specifically target ammonia for the prevention and treatment of hepatic encephalopathy in adults with cirrhosis. Cochrane Database Syst Rev 6. [crossref]

23. Zhu GQ, Shi KQ, Huang S, Wang LR, Lin YQ et.al (2015) Systematic review with network meta-analysis: the comparative effectiveness and safety of interventions in patients with overt hepatic encephalopathy. Aliment Pharmacol Ther 41: 624-35. [crossref]

\section{Citation:}

Butterworth RF (2019) Efficacy of L-Ornithine L-Aspartate for the prevention and Treatment of Hepatic Encephalopathy in Cirrhosis: An Update of the Evidence Base. J Pharmacol Pharm Res Volume 2 (6): 1-8. 